\title{
Thickness dependence of linear and quadratic magneto-optical Kerr effects in ultrathin $\mathrm{Fe}(001)$ films
}

\author{
M. Buchmeier, * R. Schreiber, D. E. Bürgler, and C. M. Schneider \\ Institut für Festkörperforschung (IFF-9) and JARA-FIT, Forschungszentrum Jülich GmbH, D-52425 Jülich, Germany
}

(Received 6 May 2008; revised manuscript received 10 December 2008; published 5 February 2009)

\begin{abstract}
Magneto-optical (MO) Kerr effect (MOKE) magnetometry is one of the most widely employed techniques for the characterization of ferromagnetic thin-film samples. Some information, such as the magnitude of coercive fields or anisotropy strengths, can be readily obtained without any knowledge of the optical and MO properties of the material. On the other hand, a quantitative analysis, which requires precise knowledge of the material's index of refraction $n$ and the MO-coupling constants $K$ and $G$, is often desirable, for instance, for the comparison of samples which are different with respect to ferromagnetic layer thicknesses, substrates, or capping layers. While the values of the parameters $n$ and the linear MO-coupling parameter $K$ reported by different authors usually vary considerably, the relevant quadratic MO-coupling parameters $G$ even for Fe are completely unknown. Here, we report on measurements of the thickness dependence $(0-60 \mathrm{~nm})$ of the linear and quadratic magneto-optical effects in epitaxial bcc-Fe(001) wedge-type samples performed at a commonly used laser wavelength of $670 \mathrm{~nm}$. By fitting the thickness dependence we are able to extract a complete set of parameters $n, K,\left(G_{11}-G_{12}\right)$, and $G_{44}$ for the quantitative description of the MOKE response of bcc-Fe( $(001)$. We find the parameters $n, K$, and $G$ to significantly differ for films thinner than about $10 \mathrm{~nm}$ as compared to those for thicker films, which is indicative of a thickness dependence of the electronic properties or of surface contributions to the MOKE. The magnitude of the quadratic magneto-optical effect is found to be about one-third of the record values reported recently for $\mathrm{Co}_{2} \mathrm{FeSi}$.
\end{abstract}

DOI: 10.1103/PhysRevB.79.064402

PACS number(s): 75.70.Ak, 78.20.Ls, 78.20.Ci, 78.20.Bh

\section{INTRODUCTION}

The magneto-optical (MO) Kerr effect (MOKE) of ferromagnetic (FM) thin films has been a field of intensive studies over the last 3 decades. This interest in MOKE was motivated by three aspects: (i) its importance as an experimental magnetometric tool, (ii) its being a means of measuring the band structure of FM materials, and (iii) its application in MO storage media. MOKE is probably the tool most widely employed for the magnetometric characterization of thin-film samples employed for spintronics. Among its most common applications are the quantitative determination of the coercivity, magnetic anisotropy, and interlayer exchange coupling from the analysis of hysteresis loops recorded with the MOKE signal. Other prominent applications are the investigation of spin dynamics in the time domain and magnetic domain imaging. The main advantages of the MOKE over other techniques are its compatibility with high magnetic fields, surface sensitivity with a typical information depth of some $10 \mathrm{~nm}$, a time resolution down to the subpicosecond regime, a reasonable spatial resolution on the order of about $0.5 \mu \mathrm{m}$, and robust and inexpensive experimental setups.

Many applications neglect the absolute magnitude of the Kerr effect, which is given by the magnitude and phase of the complex Kerr angle. Instead they describe the dependence of the normalized Kerr angle on the magnetization angle by means of adjustable response coefficients. This type of description has the advantage that it does not require any knowledge of the materials' optics, yet it is sufficient to extract a lot of information, such as the magnitude of the magnetocrystalline anisotropy of FM single-layer systems, ${ }^{1,2}$ or the antiferromagnetic interlayer exchange coupling of FM double-layer systems. ${ }^{3,4}$ On the other hand, the absolute magnitude of the complex Kerr angle provides valuable information, which can support the experimental data, and can be employed to quantitatively compare samples with, for instance, different FM layer thicknesses, substrates, or capping layers and to determine the thickness of the FM layers, the MOKE information depth, etc. However, the full quantitative MOKE information is generally not linked by simple analytic formulas to the material properties, which are the indices of refraction $n$ and the linear and quadratic MO-coupling parameters $K$ and $G$ of all involved layers. Even in ultrathin films of only some nanometers of thickness, a linear dependence of the size of the MOKE response on the FM layer thicknesses, known as additivity law-which was claimed by Qiu et al. in Ref. 5-is generally not valid. ${ }^{6}$

Therefore, a general numerical treatment of the MOKE by solving Maxwell's equations and the standard boundary conditions is indispensable for the quantitative interpretation of the Kerr angle. A prerequisite for this calculation is the precise knowledge of the optical and magneto-optical material parameters. Although spectroscopically determined values for $n$ and $K$ are available for many materials, the overall agreement of the data from different sources is often, as, for instance, in the case of bcc-Fe, ${ }^{7}$ rather poor. The strong variation in the tabulated optical constants is frequently attributed to surface contamination or oxidation of the ex situ measured samples, but thickness or quality variations of the films are also plausible. For instance, (i) in Ref. 8 the Kerr angle of $\mathrm{Fe}$ has been found to oscillate as a function of the thickness of a capping Au layer due to quantum-well states; (ii) spectroscopic MOKE data of thin Fe films show features which cannot be explained by the bulk electronic band structure of Fe (Ref. 9); and (iii) there is strong evidence for interfacial contributions in the MOKE. ${ }^{10}$ 
Moreover, the literature values are almost exclusively limited to the first-order linear MO-coupling parameter $K$. For many FM materials, e.g., from elementary Fe (Refs. 1 and 11-13) to the more complex Heusler alloy $\mathrm{Co}_{2} \mathrm{FeSi}^{14}{ }^{14}$ however, the second-order quadratic coupling constants $G$ have a comparable impact on the Kerr angle. To our knowledge the only published value of a second-order MO-coupling constant is the imaginary part of $G_{44} / K$ of $\mathrm{Ni}$ of about $\operatorname{Im}\left(G_{44} / K\right)=-0.02$ at the wavelength $\lambda=514 \mathrm{~nm}$, which was experimentally determined from Brillouin light-scattering data by Giovannini et al. ${ }^{15}$ The second-order magnetooptical parameters, which are $G_{11}, G_{12}$, and $G_{44}$ for systems with cubic symmetry, give rise to MO effects quadratic and even in the magnetization $M$. These effects are known as quadratic magneto-optical Kerr effect (QMOKE) or Voigt effect in reflection, and have recently received a lot of attention. $^{1,11-14}$ Effects quadratic in $M$ also turn out to be important in magnetization-dependent second-harmonic generation (MSHG) ${ }^{16} \mathrm{x}$-ray magnetic linear dichroism, and the closely related x-ray Voigt effect. ${ }^{17}$ All the more it is surprising that to our knowledge no one has yet determined a full set of corresponding material parameters $G$ to describe the QMOKE.

Apart from their practical significance for MOKE magnetometry, the MO-coupling parameters are also important in fundamental research. From a microscopic point of view the MO coupling is due to the interplay of the exchange interaction leading to a splitting of the bands and the spin-orbit (SO) coupling. It is therefore closely related to the magnetocrystalline anisotropy energy, which also arises due to SO coupling. The MO-coupling parameters may be seen as an important probe for the fundamental electronic interactions in FM materials; e.g., spectroscopic MOKE is a widely used standard tool to evaluate the band structure in FM metals. ${ }^{7}$ While the linear MO effect is due to first-order SO coupling, the quadratic MO coupling is thought to be caused by second-order SO coupling terms. The SO coupling is known to be altered at the interfaces and in ultrathin films, which gives rise to the well-known thickness dependence of the magnetocrystalline anisotropy energy, e.g., in Fe, ${ }^{18}$ and interfacial MOKE contributions, which have been observed by various authors. ${ }^{10}$

In this contribution we report on a magnetometric study of the magneto-optical response of bcc-Fe(001) wedge-type samples with thicknesses ranging from 0 to $60 \mathrm{~nm}$. We have determined both components of the complex Kerr angle, the Kerr rotation and the Kerr ellipticity. Effects linear and quadratic in $M$, linear magneto-optical Kerr effect (LMOKE) and QMOKE, respectively, are separated by fitting the hysteresis loops to a single domain model. The QMOKE, which is known to be anisotropic (i.e., it depends on the sample orientation with respect to the plane of incidence), has been determined for both $\mathrm{Fe}(001)[110]$ and $\mathrm{Fe}(001)[100]$ directions parallel to the plane of incidence. By fitting the thickness dependence of LMOKE and QMOKE we are able to extract a full set of Fe material parameters $n, K,\left(G_{11}-G_{12}\right)$, and $G_{44}$ at a light wavelength of $670 \mathrm{~nm}$. We find a sizable thickness dependence, which, however, seems to be mainly of nonmagnetic origin. The main effect is an increased index of refraction for Fe film thicknesses below about $10 \mathrm{~nm}$ as compared to thicker films. A large maximum absolute value of the quadratic Kerr effect (QMOKE) of $0.37 \mathrm{mrad}$ is found at about $22 \mathrm{~nm}$ Fe thickness. This value is of a comparable order of magnitude as the recently reported record QMOKE values for $\mathrm{Co}_{2} \mathrm{FeSi}^{14}$

The paper is organized as follows. In Sec. II A we describe briefly the sample preparation. Details of the experimental MOKE setup and the data recording are given in Sec. II B. Section III deals with the modeling of the MOKE and the hysteresis loops. The results are presented and discussed in Sec. IV. Finally, we summarize our results in Sec. V.

\section{EXPERIMENTAL DETAILS}

\section{A. Sample preparation and experimental MOKE setup}

Epitaxial bcc-Fe(001) (wedge)/Ag (1 nm)/Au (2 nm) have been prepared by molecular-beam epitaxy on top of a GaAs/ $\mathrm{Ag}(001)$ buffer system. The Au capping layer has been chosen to be thick enough to prevent oxidation and thin enough to be able to determine large Kerr angles. The Ag interface layer has been introduced in order to prevent a possible alloying of $\mathrm{Fe}$ and $\mathrm{Au}$. The preparation is described in detail elsewhere. ${ }^{19,20}$ All thicknesses have been precisely determined using a calibrated quartz-crystal monitor. The Fe thickness has been varied continuously between 0 and $8 \mathrm{~nm}$ for sample A and stepwise for sample B with discrete $\mathrm{Fe}$ thicknesses of $5,8,12,18,24,32,44$, and $60 \mathrm{~nm}$.

\section{B. MOKE setup}

The MOKE measurements were performed using light from a Toshiba TOLD9231M multimode laser diode with a wavelength of $670 \mathrm{~nm}$ and a spectral half-width of less than $2 \mathrm{~nm}$. An in-plane magnetic field with a maximum strength of $0.7 \mathrm{~T}$ is generated by a Broker electromagnet with FM yoke and measured with a Hall probe. The sample is mounted on a manually rotatable holder with an angle scale, which allows a determination of the angle with respect to the plane of incidence with a precision of about $\pm 2^{\circ}$.

The optical setup consists of a light beam with its plane of incidence parallel to the external field direction illuminating the sample under an angle of incidence of $15^{\circ}$ with respect to the sample normal (see Fig. 1). The incident light is polarized in $\hat{p}$ direction (electric field component in the plane of incidence) employing a rotatable Glan-Taylor-type polarizer. The phase of the reflected light is modulated at a frequency $f=50 \mathrm{kHz}$ with a HINDS PEM-90 photoelastic modulator (PEM) at diagonal modulation axis (rotated by $45^{\circ}$ with respect to the $\hat{p}$ direction) and at a retardation of $90^{\circ}$. A quarter wave plate with its retardation axis parallel to the $\hat{p}$ direction can be placed between sample and PEM. Consecutively the light passes through an analyzer of the same type as the polarizer, but oriented in $\hat{s}$ direction. The light intensity is then converted into an electrical voltage by a homemade diode detector. In order to avoid possible multiple reflections between the sample and the optical elements in the reflected beam path, which might impair the data by parasite signals, a diaphragm with a diameter of about $2 \mathrm{~mm}$ is placed right after the sample. Analyzer, PEM, and quarter wave plate are 


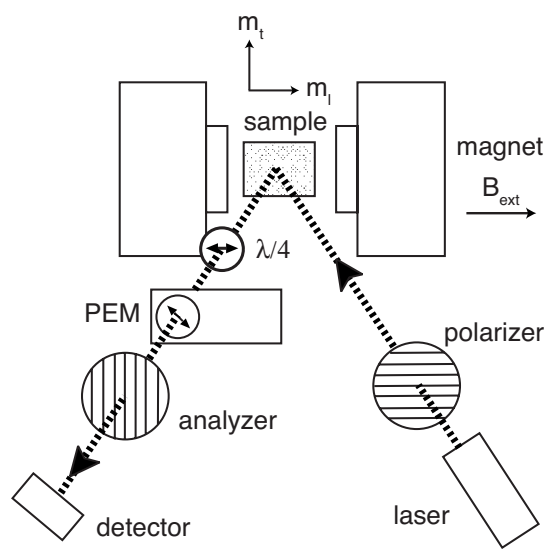

FIG. 1. Experimental MOKE setup. The quarter wave plate is introduced to measure the Kerr rotation $\theta$ instead of the Kerr ellipticity $\epsilon$. For clarity, the diaphragm is not shown.

slightly tilted with respect to the optical axis so that the light reflected back to the sample is blocked by the diaphragm.

With this setup the small $f(50 \mathrm{kHz})$ component determined with a lock-in amplifier is to first order proportional to the $\hat{p}$ ellipticity $\epsilon$ times the reflected intensity, while the much larger $2 f(100 \mathrm{kHz})$ and $\mathrm{dc}$ components (the latter is recorded simultaneously with a multimeter) are to first order proportional to the reflected intensity alone ${ }^{21} \mathrm{By}$ introducing a quarter wave plate between sample and PEM, we are able to measure the $\hat{p}$ rotation $\theta$ instead of the ellipticity. The two Kerr angle components $(\theta$ and $\epsilon$ ) are calculated by dividing the measured $f$ component by the $2 f$ component.

As the amplification factor of the detector is frequency dependent, the measured Kerr angle has to be calibrated. For this purpose, we have used a thick Au sample and turned the analyzer out of the $\hat{p}$ direction by an angle $\Psi$ while recording the ellipticity and rotation signal. The data are then adjusted such that the measured dependence of the resulting rotation $\theta$ and ellipticity $\epsilon$ as a function of the polarizer angle $\Psi$ matches the theoretical relationship

$$
\theta(\Psi)+i \epsilon(\Psi)=\frac{r_{s s}}{r_{p p}} \sin (\Psi),
$$

where $r_{s s}$ and $r_{p p}$ are the diagonal Fresnel reflection coefficients. A value $r_{s s} / r_{p p}=1.0003-0.0379 i$ has been calculated using the Fresnel formulas and taking the literature value ${ }^{22}$ $n=0.100+3.653 i$ for the index of refraction of $\mathrm{Au}$ at our laser wavelength. We have performed the calibration procedure for both the real and imaginary parts of Eq. (1), i.e., with and without quarter wave plate, and find excellent agreement for the calibration factors (less than $2 \%$ difference). This corroborates that the calibration works properly and possible detriments of the measurement by multiple reflections are indeed well under control.

\section{NUMERICAL MODELING OF THE MOKE AND HYSTERESIS LOOPS}

The optical and MO material properties can be described by the permittivity tensor $\epsilon_{i j}$, which can be expanded in a power series of the Cartesian direction cosines $m_{i}$ of the magnetization $\vec{M}$ :

$$
\epsilon_{i j}(\vec{M})=\epsilon_{i j}^{(M=0)}+K_{i j k} m_{k}+G_{i j k s} m_{k} m_{s}+\cdots .
$$

The numbers of independent linear and quadratic MOcoupling constants, $K_{i j k}$ and $G_{i j k s}$, respectively, are reduced by the symmetry of the crystal and the Onsager principle $\epsilon_{i j}(\vec{M})=\epsilon_{i j}(-\vec{M})$. For cubic symmetry the permittivity tensor is completely defined by five quantities: the nonmagnetic part of the permittivity $\epsilon^{(M=0)}=n^{2}$, which is given by the square of the index of refraction $n$; the linear MO-coupling constant $K=K_{i j k}$; and the three independent quadratic MOcoupling parameters $G_{i i i i}=G_{11}, G_{i i j j}=G_{12}$, and $G_{i j i j}=G_{44}$. Instead of the linear MO coupling $K$, the Voigt parameter $Q$ $=i K / \epsilon^{(M=0)}$ is frequently used. The complex Kerr angle, which is a measure of the magnitude of the MOKE signal, can be calculated using the standard $4 \times 4$ matrix formalism as explained in Refs. 23-25. Our open-source computer program developed for the calculation of the MOKE can be downloaded from Ref. 26.

The small complex Kerr angle is generally defined as the off-diagonal Fresnel reflection coefficients divided by the diagonal ones. For instance, for incident $p$-polarized light: $\Phi_{p}=E_{s}^{\mathrm{refl}} / E_{p}^{\mathrm{inc}}=r_{p s} / r_{p p}=\theta+i \epsilon^{27}$ We would like to point out that this definition is insufficient as it does by no means define the sign of the Kerr angle, which depends on the choice of coordinate system, relative orientation of the $\hat{p}$ and $\hat{s}$ directions, the in-plane wave vector of the light, and the sign of the $\omega t$ term in the exponent of the wave function, i.e., the time convention. The sign convention in magneto-optics is indeed a long-standing problem; i.e., different authors report different signs for the complex Kerr and Faraday rotation angles. ${ }^{7}$ Even worse hardly any paper gives a clear definition of the employed sign conventions. Therefore, we will here briefly define the convention used in this paper. The orientation of the coordinate system is defined as depicted in Fig. 1 with positive $m_{l}$, which is also the direction of a positive external field pointing to the right; $m_{t}$ pointing up; and $k_{\|}$, which is the direction of the in-plane light wave vector, pointing to the left when looking onto the sample. The $\hat{p}$ and $\hat{s}$ directions of the incident and reflected beams are chosen to be parallel to $m_{l}$ and $m_{t}$, respectively, for perpendicular incidence, and a time convention of $\vec{k} \vec{r}-\omega t$ is assumed. Using this convention a positive Kerr rotation corresponds to a rotational vector pointing in the propagation direction of the reflected light; i.e., the polarization vector ( $\hat{p}$ direction) is rotated in clockwise direction when looking in the direction of the reflected beam. For a $60 \mathrm{~nm}$ Fe film the Kerr rotation due to a positive $m_{l}$ results in a negative Kerr rotation $\theta$ and a positive Kerr ellipticity $\epsilon$. From an experimental point of view, the negative Kerr rotation is equivalent in sign to a counterclockwise turn (looking in the direction of the incident beam) of the polarizer and the positive ellipticity $\epsilon$ to a clockwise turn of the polarizer out of the $\hat{p}$ direction, respectively.

It is convenient to expand the Kerr angle $\Phi$ as a function of the directional cosines of the magnetization vector $\vec{M}$ (Ref. 13); e.g., for in-plane magnetization, 


$$
\Phi=\sum_{\text {layers } i}\left[l_{i} m_{l, i}+q_{1, i} m_{l, i} m_{t, i}+q_{2, i} m_{t, i}^{2}+O\left(m^{3}\right)\right],
$$

where $l_{i}$ are the longitudinal (LMOKE) response coefficients and $q_{1, i}$ and $q_{2, i}$ are the quadratic response coefficients. Similar relations hold for the more general case including out-ofplane magnetization, the Faraday effect, and even the calculation of Brillouin light-scattering intensities, ${ }^{28}$ which are closely related to the MOKE problem. Note that some authors use a different form for the second quadratic term, namely, $q_{2}\left(m_{l}^{2}-m_{t}^{2}\right)$, which is equivalent to our term if the magnetization is in a single domain state except for a factor of -2 and a constant offset of 1 . In the case of a multidomain state this kind of description does not generally hold as additional significant $\mathrm{MO}$ effects due to the magnetization gradient ${ }^{29}$ can be present. The longitudinal coefficients $l_{i}$ stem from the linear MO-coupling parameter $K$ alone and are known to be isotropic, i.e., independent on the sample orientation, as long as the FM layers have cubic symmetry. By contrast, the quadratic coefficients are due to a combined effect of the linear and quadratic MO couplings and are anisotropic; i.e., they depend on the relative orientation of the sample with respect to the plane of incidence. For cubic systems the resulting $q$ coefficients have been found to have the form $^{13}$

$$
\begin{gathered}
q_{1}=q_{001}+\left(q_{011}-q_{001}\right) \sin ^{2}(2 \gamma), \\
q_{2}=\frac{1}{2}\left(q_{011}-q_{001}\right) \sin (4 \gamma),
\end{gathered}
$$

where $\gamma$ is the angle between the in-plane component of the light wave vector and an in-plane $\mathrm{Fe}[001]$ direction (positive $\gamma$ corresponds to a counterclockwise sample rotation in Fig. 1), and $q_{001}$ and $q_{011}$ are QMOKE constants for the planes of incidence parallel to the [001] and [011] directions, respectively. The anisotropy of the QMOKE stems from the symmetry of the effective second-order MO-coupling tensor $G_{i j k s}$, which is closely related to the symmetry of the crystal. For $\mathrm{Fe}(001)$ with in-plane magnetization, $\left(G_{11}-G_{12}\right)$ and $2 G_{44}$ are the relevant second-order MO-coupling parameters for the [011] $\left(\gamma=45^{\circ}\right)$ and [001] directions $\left(\gamma=0^{\circ}\right)$, respectively. The parameter $\Delta G=\left(G_{11}-G_{12}\right)-2 G_{44}$ is a measure of the anisotropy strength. ${ }^{13}$

The $q_{1}$ coefficients have an isotropic and an anisotropic contribution. They depend on the sum of $-K^{2} / n^{2}$ and $G_{11}$ $-G_{12}-\Delta G \cos ^{2}(2 \gamma) \cdot{ }^{13}$ In some cases the first term is much smaller than the second term; however for our system and experimental setup both terms have a comparable order of magnitude. Therefore, errors in the determination of $n$ and $K$ affect the accuracy of the $G$ values. A wrong sign of the $q_{1}$ value will not simply lead to wrong signs, but to wrong values of the second-order MO-coupling constants. On the other hand, the $q_{2}$ coefficients stem purely from the $G$ parameters, namely, from $\Delta G / 2 \sin (4 \gamma),{ }^{13}$ and vanish if the plane of incidence is parallel to the symmetry directions [001] and [011].

The response coefficients can be determined experimentally - at least for single-layer systems-with a suitable setup, for instance, by rotating the field. ${ }^{13,30}$ They

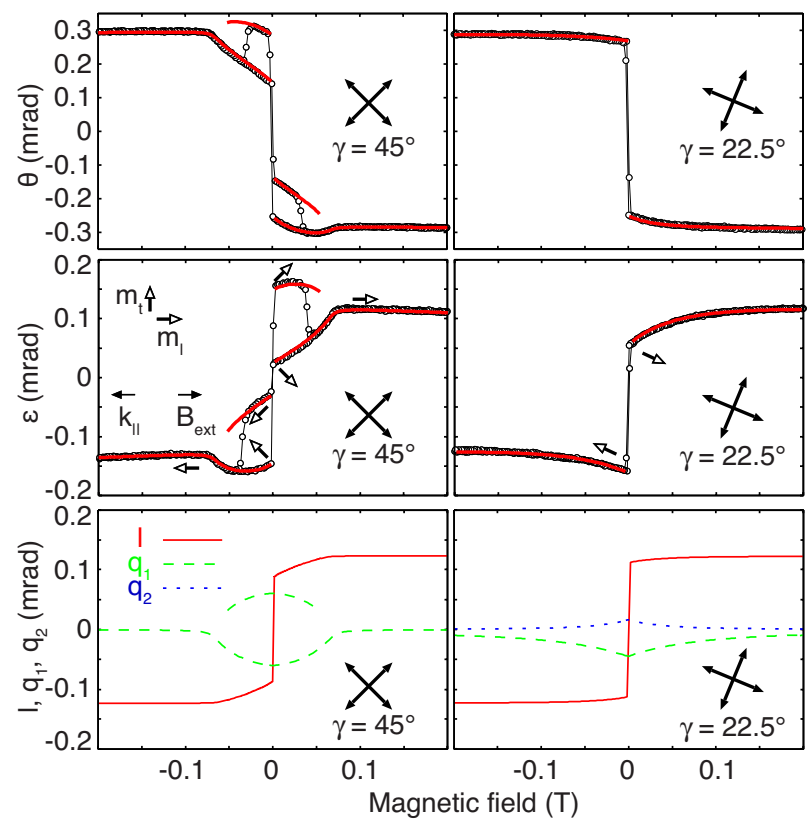

FIG. 2. (Color online) Field dependences of measured (connected open circles) and calculated (lines) Kerr ellipticities (center graphs) and Kerr rotations (top graphs) for a $60 \mathrm{~nm} \mathrm{Fe} \mathrm{film.} \mathrm{The}$ bottom graphs show the components corresponding to the $l, q_{1}$, and $q_{2}$ terms of the Kerr ellipticity loops. The left curves correspond to a sample orientation with hard axis parallel to external field $(\gamma$ $\approx 45^{\circ}$ ), while for the right curves the field is parallel to an intermediate direction $\left(\gamma \approx 22.5^{\circ}\right)$. The field is swept first from positive to negative values and then back. The magnetization alignments are shown with short arrows; the easy axis directions of the magnetocrystalline anisotropy are shown with crossed long arrows.

can also be calculated numerically from the optical and MO material parameters $n, K$, and $G$. As our MOKE setup does not allow for a field rotation, we choose here the alternative approach of analyzing remagnetization loops recorded at different sample orientations.

Typical MOKE loops for a $60 \mathrm{~nm}$ Fe film are shown in Fig. 2. The experimental Kerr rotation (top graphs) and ellipticity (center graphs) are plotted with connected open circles. We have determined the MOKE response coefficients $l, q_{1}$, and $q_{2}$ by fitting experimental remagnetization loops to a single domain model taking into account the sample orientation $\gamma$ and the cubic anisotropy parameter $K_{c} / M_{s}$, and describing the Kerr angle via Eqs. (3)-(5). While the left loops recorded at an angle $\gamma \approx 45^{\circ}$ (i.e., with field parallel to a hard [011] direction) depend on $l$ and $q_{011}$, the right loops are recorded at $\gamma \approx 22.5^{\circ}$ and, therefore, depend on $l$ and both $q_{011}$ and $q_{001}$. Thus, a simultaneous fitting of the loops for both orientations yields a full set of MOKE response coefficients $l, q_{001}$, and $q_{011}$.

\section{RESULTS AND DISCUSSION}

\section{A. Linear MOKE}

The thickness dependence of the experimental linear MOKE extracted from the hard axis loops is marked in Fig. 3 by circles and triangles for samples A and B, respectively. 


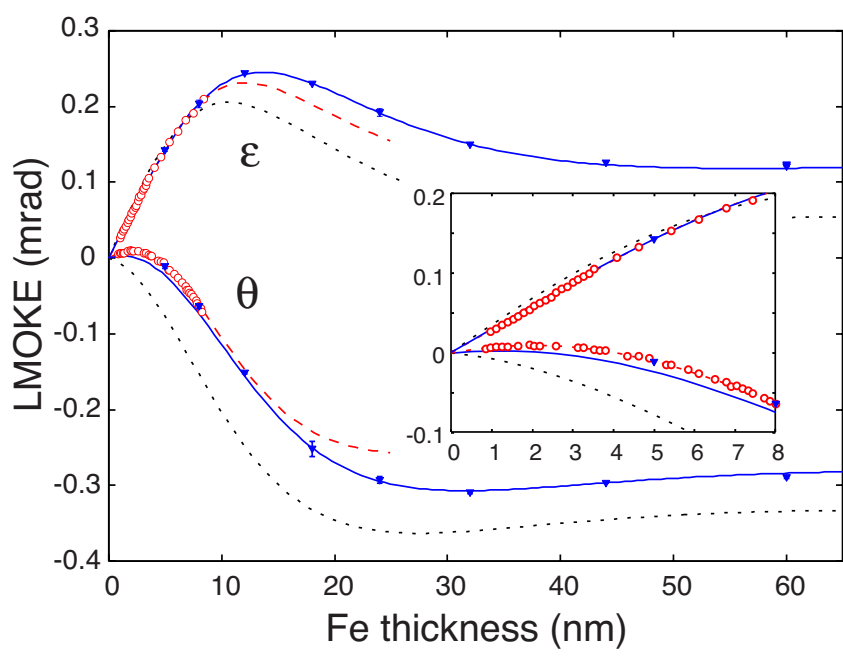

FIG. 3. (Color online) Thickness dependences of the measured (circles and triangles for samples A and B, respectively) and calculated (lines) linear ellipticities $\epsilon$ (upper curves) and rotations $\theta$ (lower curves). Error bars are comparable to the symbol size. Dotted curves result from a calculation with material parameters from literature. A fit to the data of sample A with thickness range of $0-8$ $\mathrm{nm}$ (dashed curves) yields a clearly different behavior from a fit to the data of sample B with a larger thickness range of 5-60 nm (solid curves). Inset: Magnification of the low-thickness region.

The data for thicknesses of 5 and $8 \mathrm{~nm}$ measured for both samples are in excellent agreement, indicating that the sample qualities and thickness calibrations of samples A and $\mathrm{B}$ are very similar. The upper and lower curves correspond to the imaginary and real parts of the Kerr angle (ellipticity $\epsilon$ and rotation $\theta$ ), respectively. In agreement with the results of previous publications on similar systems, ${ }^{5}$ the Kerr ellipticity increases linearly with the film thickness below $5 \mathrm{~nm}$, which is indicative of the law of additivity of the MOKE effect size being valid in this regime. On the other hand, there is no such linear behavior for the Kerr rotation, which changes sign at about $4 \mathrm{~nm}$, meaning that the additivity does not hold for the Kerr rotation. This breakdown of the additivity law is due to the dominant imaginary component of the Kerr angle found for ultrathin $\mathrm{Fe}$ layers on noble-metal substrates. In contrast, on semiconducting substrates, e.g., GaAs, the Kerr angle of $\mathrm{Fe}$ is mainly real and the additivity holds for the rotation, but not for the ellipticity. ${ }^{6}$ For thicker layers, the phase of the electromagnetic wave inside the $\mathrm{Fe}$ layer changes due to the real part of the perpendicular wave-vector component $k_{\perp}$ and, therefore, gives rise to negative Kerr ellipticity contributions coming from Fe layers buried deeper than about $12 \mathrm{~nm}$, where the slope changes sign. On the other hand, the imaginary part of the wave vector $k_{\perp}$ leads to a decreasing intensity of the electromagnetic wave with increasing depth inside the film, which determines the information depth of about $40 \mathrm{~nm}$, where the slope begins to asymptotically flatten.

The thickness dependence calculated using literature values of the indices of refraction from Refs. 22 and 31, $n_{\mathrm{Ag}}$ $=0.27+4.66 i, n_{\mathrm{Au}}=0.10+3.65 i$, and $n_{\mathrm{Fe}}=3.57+4.02 i$, and the linear MO coupling from Ref. 32, $Q=0.0437+0.0040 i$, is plotted as dotted lines. As found earlier by Qiu et al. ${ }^{5}$ the material constants from literature approximately reproduce the Kerr ellipticity, which is insensitive to small phase changes of $Q$. However, the literature data fail to describe the Kerr rotation.

We have fitted our experimental data employing the full $4 \times 4$ matrix formalism ${ }^{23,24}$ using fixed indices of refraction for $\mathrm{Ag}$ and $\mathrm{Au}$ from literature as specified above, and treating the index of refraction and the MO coupling of $\mathrm{Fe}$ as free parameters. The dashed and solid curves in Fig. 3 correspond to the data of samples A and B, respectively. The fit results are listed in Table I. It turns out that a satisfactory fit over the whole thickness range with only one thickness-independent set of material parameters is impossible. The fit to the data of sample A with smaller thicknesses results in a significantly about $10 \%$ larger index of refraction with different phase as compared to the thicker sample B. On the other hand, the MO-coupling parameter $Q$ mainly differs in phase by about $10^{\circ}$, while the absolute values are in rather good agreement within less than $3 \%$ difference. Thus, it seems that the difference between thin and thick Fe layers is mainly of optic rather than of magneto-optic origin.

Our value of the index of refraction of $\mathrm{Fe}$ determined from the data of the thicker sample $\mathrm{B}, n_{\mathrm{Fe}}=3.53+3.72 i$, compares reasonably well with the value $n=3.57+4.02 i$ of Yolken and Kruger. ${ }^{31}$ While the real part is in excellent agreement, our imaginary part is about $7 \%$ smaller, which is probably within the range of the systematic experimental errors. On the other hand, our value for the linear MO coupling $Q$ for sample B is significantly by about $20 \%$ smaller and has a phase difference of about $17^{\circ}$ compared to the data of Krinchik and Artemev. ${ }^{32}$

The curve fitted to the data from the thinner sample departs from the experimental data at about $15 \mathrm{~nm}$, which corresponds to approximately half the penetration depth of the light. This circumstance might hint at an improper description of the optical properties of the $\mathrm{Ag}$ substrate as a reason

TABLE I. Indices of refraction $(n)$ and linear $(Q)$ and quadratic $\left(G_{11}-G_{12}\right.$ and $\left.G_{44}\right)$ MO-coupling constants derived from data of samples A and B.

\begin{tabular}{lccc}
\hline \hline Parameter & Sample A $(0-8 \mathrm{~nm})$ & Sample B $(5-60 \mathrm{~nm})$ & Literature $^{\mathrm{a}}$ \\
\hline$n$ & $4.06 \pm 0.03+(3.85 \pm 0.03) i$ & $3.53 \pm 0.03+(3.72 \pm 0.03) i$ & $3.57+4.02 i$ \\
$Q$ & $0.0331 \pm 0.0002-(0.0127 \pm 0.0002) i$ & $0.0356 \pm 0.0004-(0.0074 \pm 0.0003) i$ & $0.0437+0.004 i$ \\
$G_{11}-G_{12}$ & $-0.0544 \pm 0.0005-(0.0287 \pm 0.0005) i$ & $-0.0358 \pm 0.0002-(0.0382 \pm 0.0002) i$ & \\
$G_{44}$ & & $-0.0117 \pm 0.0003-(0.0349 \pm 0.0003) i$ & \\
\hline \hline
\end{tabular}

${ }^{\mathrm{a} R e f e r e n c e s} 22$ and 31. 


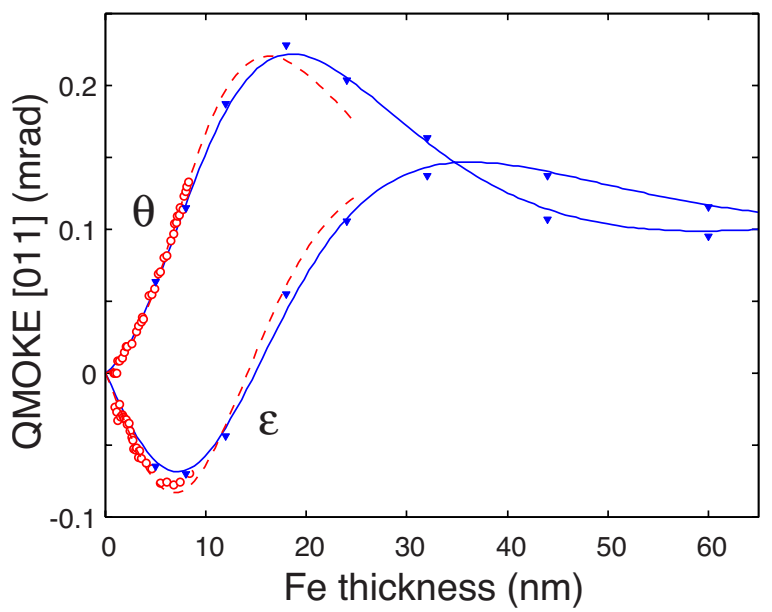

FIG. 4. (Color online) Thickness dependences of the measured (circles and triangles for samples A and B, respectively) and calculated (lines) QMOKEs for the Fe[011] direction (hard axis) parallel to the plane of incidence.

for the apparent thickness dependence of the index of refraction of Fe. The substrate mainly influences the Kerr angle for Fe thicknesses below $15 \mathrm{~nm}$ as the light reflected from the substrate can reach the sample surface. In order to test this conjecture, we have additionally fitted the data with the indices of refraction of the substrate and capping layers as free parameters. However, we could not substantially improve the overall quantitative agreement of the fits. Therefore, improper optical parameters of the nonmagnetic layers can be ruled out as a reason for the encountered thickness dependence of the optical Fe properties. Possible explanations for the thickness dependence are: (i) the tensile strain of the $\mathrm{Fe}$ due to the small lattice mismatch of $0.7 \%$ between $\mathrm{Fe}$ and the $\mathrm{Ag}$ substrate leading to an anisotropic permittivity tensor; (ii) a relaxation of the lattice constant of Fe for larger thicknesses resulting in a thickness dependence of the optic properties of the Fe layer; (iii) altered electronic properties of the thin Fe layer due to the proximity to the noble-metal substrate and the capping layers, which can have a sizable influence, ${ }^{8,10}$ and (iv) interfacial MOKE contributions, ${ }^{10}$ which have been neglected in the theoretical description.

\section{B. Quadratic MOKE}

The thickness dependences of the QMOKE coefficients $q_{1}$ for the hard axis $(\mathrm{Fe}[011]$ direction) and the easy axis $(\mathrm{Fe}[001]$ direction) configurations are plotted in Figs. 4 and 5 , respectively. In contrast to LMOKE, in this case the real part (Kerr rotation $\theta$ ) is the quantity which depends linearly on thickness for ultrathin Fe layers, while the slope of the imaginary part (Kerr ellipticity $\epsilon$ ) changes sign at approximately 7 and $2 \mathrm{~nm}$ for the [011] and [001] configurations, respectively. Thus, taking into account the significant QMOKE contribution the additivity holds for neither component of the Kerr angle. It should, however, be noted that the additivity holds for the complex Kerr angle if the layers are sufficiently thin.

The extracted relevant second-order MO-coupling constants, $G_{11}-G_{12}$ and $G_{44}$, for the hard and easy axis configu-

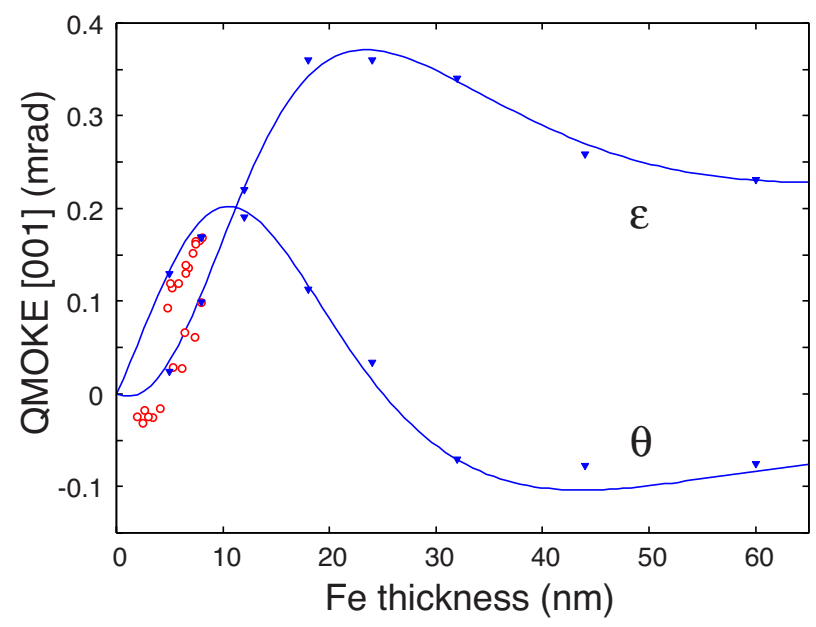

FIG. 5. (Color online) Thickness dependences of the measured (circles and triangles for samples A and B, respectively) and calculated (line) QMOKEs for the Fe[001] direction (easy axis) parallel to the plane of incidence.

rations are listed in Table I. They have been determined by fitting the data taking into account the $n$ and $Q$ parameters from the fits to the LMOKE data (Fig. 3). The determination of the $q_{001}$ parameter depends on both the values of $q_{011}$ and $l$ [see Eq. (3)]. Therefore, the propagation of errors results in a significantly poorer quality of the experimental data. As a consequence we could not reliably determine the $G_{44}$ parameter for sample A.

We find maximum absolute values $\left|q_{1}\right|$ of the QMOKE at about $22 \mathrm{~nm}$ of 0.23 and $0.37 \mathrm{mrad}$ for the hard and easy axis configurations, respectively. These values should be compared to the record QMOKE value of about $1.05 \mathrm{mrad}$ (Ref. 33) recently found in $\mathrm{Co}_{2} \mathrm{FeSi}$ alloys in Ref. 34. These comparable orders of magnitudes of the QMOKEs of $\mathrm{Fe}$ and $\mathrm{Co}_{2} \mathrm{FeSi}$ imply that the maximum QMOKE value of $\mathrm{Fe}$ in the visible wavelength region might be even larger than that of $\mathrm{Co}_{2} \mathrm{FeSi}$ as both materials are expected to have distinct frequency dependences resulting from their electronic band structures.

In Fig. 2 of Ref. 13 Postava et al. gave the dependence of LMOKE and QMOKE contributions on sample orientation for a $50-\mathrm{nm}$-thick bcc-Fe(001) sample capped with $1.5 \mathrm{~nm}$ $\mathrm{Pd}$ and measured at an incident angle of $3.25^{\circ}$. Based on our fitted optical and MO material constants we have calculated the $\operatorname{Re}(l), \operatorname{Re}\left(q_{001}\right)$, and $\operatorname{Re}\left(q_{011}\right)$ constants for the sample structure and experimental configuration of Ref. 13 and assuming $n_{\mathrm{Pd}}=1.87+4.44 i$. We find a value of $\operatorname{Re}(l)=$ -0.066 mrad, which is comparable but about $20 \%$ smaller than the value of $\operatorname{Re}(l) \approx-0.09 \mathrm{mrad}$ (Ref. 33) determined by Postava et $a l .{ }^{13}$ On the other hand, our QMOKE data differ more distinctly, although they are of a similar order of magnitude. While we find $\operatorname{Re}\left(q_{011}\right)=0.079$, which is about $30 \%$ larger than the value $\operatorname{Re}\left(q_{011}\right) \approx 0.06$ determined by Postava et al. ${ }^{13}$ our value for $\operatorname{Re}\left(q_{001}\right)=-0.073$ is about a factor of 2 smaller than $\operatorname{Re}\left(q_{001}\right) \approx-0.16$ determined by Postava et al. ${ }^{13}$ This difference of the QMOKE is clearly larger than the experimental errors caused by uncertainties in the layers thicknesses, the optical properties of the capping layers, and the calibration of the MOKE setup. Although 
both the sample of Postava et al. ${ }^{13}$ and our samples are epitaxial bcc-Fe(001), a possible explanation could be a strong structural dependence of the QMOKE as described in Ref. 14 for $\mathrm{Co}_{2} \mathrm{FeSi}$.

\section{CONCLUSIONS}

The thickness dependence of the linear and quadratic MOKEs of wedge-type thin $\mathrm{Fe}(001)$ films magnetized in the sample plane has been measured. Good quantitative agreement of the experimental data with calculations assuming bulk-type optic and MO material constants indicates that the thickness dependence of the MOKE is mainly due to bulktype magneto-optical coupling. On the other hand, we found a sizable departure from theory for thicknesses below about $10 \mathrm{~nm}$. This can be explained by thickness-dependent optic and MO material parameters, which are possibly due to MO surface effects or thickness-dependent features of the band structure, e.g., quantum-well states. By fitting the experimental data to results of a numerical model, we extracted a complete set of material constants $n, K,\left(G_{11}-G_{12}\right)$, and $G_{44}$ for the quantitative description of the MOKE of bcc-Fe(001) at the laser frequency employed. These second-order MOcoupling constants of $\mathrm{Fe}$ are comparable to the first-order constants and, thus, of general significance for the theoretical description of the MOKE. The index of refraction $n$ is in excellent agreement and the linear MO-coupling constants $K$ agree reasonably with previous works. In contrast, a comparison of the second-order constants with earlier QMOKE data from Postava et al. ${ }^{13}$ demonstrates a remarkable difference. This suggests that the anisotropic second-order MO coupling might strongly depend on the sample properties.

\section{ACKNOWLEDGMENTS}

The authors would like to thank K. Postava for helpful discussions.
*Present address: Institut für Angewandte Physik, Corrensstrasse 2-4, Westfälische Wilhelms-Universität Münster, D-48149 Münster, Germany; m.buchmeier@uni-muenster.de

${ }^{1}$ S. Yan, R. Schreiber, P. Grünberg, and R. Schäfer, J. Magn. Magn. Mater. 210, 309 (2000).

${ }^{2}$ D. Berling, S. Zabrocki, R. Stephan, G. Garreau, J. L. Bubendorff, A. Mehdaoui, D. Bolmont, P. Wetzel, C. Pirri, and G. Gewinner, J. Magn. Magn. Mater. 297, 118 (2006).

${ }^{3}$ R. R. Gareev, D. E. Bürgler, M. Buchmeier, R. Schreiber, and P. Grünberg, J. Magn. Magn. Mater. 240, 235 (2002).

${ }^{4}$ M. Buchmeier, Ph.D. thesis, Universität zu Köln, 2003 (http:// kups.ub.uni-koeln.de/volltexte/2003/677/).

${ }^{5}$ Z. Q. Qiu, J. Pearson, and S. D. Bader, Phys. Rev. B 45, 7211 (1992).

${ }^{6}$ M. Nyvlt, M. Przybylski, J. Grabowski, and J. Kirschner, J. Appl. Phys. 98, 033516 (2005).

${ }^{7}$ P. M. Oppeneer, in Handbook of Magnetic Materials, edited by K. H. J. Buschow (Elsevier Science/North-Holland, Amsterdam, 2001), Vol. 13, p. 229.

${ }^{8}$ Y. Suzuki, T. Katayama, P. Bruno, S. Yuasa, and E. Tamura, Phys. Rev. Lett. 80, 5200 (1998).

${ }^{9}$ J. Grondilova, M. Rickart, J. Mistrik, K. Postava, S. Visnovsky, T. Yamaguchi, R. Lopusnik, S. O. Demokritov, and B. Hillebrands, J. Appl. Phys. 91, 8246 (2002).

${ }^{10}$ J. Hamrle, M. Nyvlt, S. Visnovsky, R. Urban, P. Beauvillain, R. Megy, J. Ferre, L. Polerecky, and D. Renard, Phys. Rev. B 64, 155405 (2001).

${ }^{11}$ K. Postava, H. Jaffres, A. Schuhl, F. Nguyen Van Dau, M. Goiran, and A. R. Fert, J. Magn. Magn. Mater. 172, 199 (1997).

${ }^{12}$ R. M. Osgood, B. M. Clemens, and R. L. White, Phys. Rev. B 55, 8990 (1997).

${ }^{13}$ K. Postava, D. Hrabovsky, J. Pistora, A. R. Fert, S. Visnovsky, and T. Yamaguchi, J. Appl. Phys. 91, 7293 (2002).

${ }^{14}$ J. Hamrle, S. Blomeier, O. Gaier, B. Hillebrands, H. Schneider, G. Jakob, K. Postava, and C. Felser, J. Phys. D 40, 1563 (2007).

${ }^{15}$ L. Giovannini, R. Zivieri, G. Gubbiotti, G. Carlotti, L. Pareti, and G. Turilli, Phys. Rev. B 63, 104405 (2001).

${ }^{16}$ E. D. Mishina, A. I. Morosov, A. V. Mishina, V. Moshnyaga, L.
Sudheendra, K. Samwer, and Th. Rasing, Phys. Rev. B 75 064401 (2007).

${ }^{17}$ H. C. Mertins, S. Valencia, A. Gaupp, W. Gudat, P. M. Oppeneer, and C. M. Schneider, Appl. Phys. A: Mater. Sci. Process. 80, 1011 (2005).

${ }^{18}$ R. J. Hicken, S. J. Gray, A. Ercole, C. Daboo, D. J. Freeland, E. Gu, E. Ahmad, and J. A. C. Bland, Phys. Rev. B 55, 5898 (1997).

${ }^{19}$ D. E. Bürgler, C. M. Schmidt, J. A. Wolf, T. M. Schaub, and H.-J. Güntherodt, Surf. Sci. 366, 295 (1996).

${ }^{20}$ D. E. Bürgler, C. M. Schmidt, D. M. Schaller, F. Meisinger, R. Hofer, and H.-J. Güntherodt, Phys. Rev. B 56, 4149 (1997).

${ }^{21}$ M. Buchmeier, diploma thesis, Universität zu Köln, 1999 (http:// hdl.handle.net/2128/2892).

${ }^{22}$ Handbook of Chemistry and Physics, 82 nd ed., edited by D. R. Lide (CRC, Cleveland, 2002).

${ }^{23}$ P. Yeh, Surf. Sci. 96, 41 (1980).

${ }^{24}$ S. Visnovsky, Czech. J. Phys. 41, 663 (1991).

${ }^{25}$ J. Zak, E. R. Moog, C. Liu, and S. D. Bader, Phys. Rev. B 43, 6423 (1991).

${ }^{26} \mathrm{http}: / /$ hdl.handle.net/2128/2605

${ }^{27}$ Note that the indexing order of coefficients of the reflection matrix $r_{i j}$ differs from author to author. Here we use the same convention as in Ref. 23, where $E_{s}^{\mathrm{refl}}=r_{p s} E_{p}^{\text {inc }}$.

${ }^{28}$ M. Buchmeier, H. Dassow, D. E. Bürgler, and C. M. Schneider, Phys. Rev. B 75, 184436 (2007).

${ }^{29}$ R. Schäfer and A. Hubert, Phys. Status Solidi A 118, 271 (1990).

${ }^{30}$ R. Mattheis and G. Quednau, J. Magn. Magn. Mater. 205, 143 (1999).

${ }^{31}$ H. T. Yolken and J. Kruger, J. Opt. Soc. Am. 55, 842 (1965).

${ }^{32}$ G. S. Krinchik and V. A. Artemev, Sov. Phys. JETP 26, 1080 (1968).

${ }^{33}$ References 13 and 14 give the size of QMOKE originating from $m_{l} m_{t}$ in saturation, which is a factor of 2 smaller than our definition of the $q_{1}$ parameter.

${ }^{34}$ J. Hamrle, S. Blomeier, O. Gaier, B. Hillebrands, H. Schneider, G. Jakob, B. Reuscher, A. Brodyanski, M. Kopnarski, K. Postava, and C. Felser, J. Phys. D 40, 1558 (2007). 